\title{
Downregulation of LINC01140 is associated with adverse features of breast cancer
}

\author{
DEHENG LI ${ }^{1,2^{*}}$, LIANGDONG $\mathrm{LI}^{1,2^{*}}$, YIQUN CAO ${ }^{1,2}$ and XIN CHEN ${ }^{1,2}$ \\ ${ }^{1}$ Department of Neurosurgery, Fudan University Shanghai Cancer Center; ${ }^{2}$ Department of \\ Oncology, Shanghai Medical College, Fudan University, Shanghai 200032, P.R. China
}

Received May 12, 2019; Accepted November 1, 2019

DOI: $10.3892 / \mathrm{ol} .2019 .11147$

\begin{abstract}
Breast cancer (BC) is one of the most dangerous malignant diseases among women. A growing amount of evidence has suggested that long non-coding RNAs participate in the development and progression of $\mathrm{BC}$ and may potentially serve as therapeutic targets or prognostic markers for the disease. A previous study demonstrated that long intergenic non-protein coding RNA 01140 (LINC01140) was prominently correlated with overall survival in patients with gastric cancer. However, the function of LINC01140 in BC has not yet been elucidated. Therefore, the present study aimed to investigate the roles and molecular mechanisms underlying LINC01140 in BC. LINC01140 expression in 1,085 breast cancer patients and 291 healthy subjects was analyzed from the Gene Expression Profiling Interactive Analysis website. The association between LINC01140 expression and T stages, LINC01140-related biological pathways, and the correlation between LINC01140 expression genes were also analyzed in 825 patients with BC through the cBioPortal database. The present study demonstrated that LINC01140 expression was significantly decreased in the tumor samples compared with normal samples in patients with $\mathrm{BC}(\mathrm{P}<0.05)$. The present study revealed that LINC01140 expression was significantly decreased in the T4 stage compared with $\mathrm{T} 1, \mathrm{~T} 2$ or T3 stage $(\mathrm{P}<0.01)$. In addition, high expression levels of LINC01140 predicts longer relapse-free survival probability in patients with BC. It was also observed that LINC01140 participates in a variety of biological pathways, particularly in the epithelial-to-mesenchymal transition. The co-expression relationship
\end{abstract}

Correspondence to: Professor Yiqun Cao or Professor Xin Chen, Department of Neurosurgery, Fudan University Shanghai Cancer Center, 270 Dong'an Road, Xuhui, Shanghai 200032, P.R. China

E-mail: fudancaoyiqun@163.com

E-mail: 49556818@qq.com

*Contributed equally

Key words: breast cancer, long non-coding RNA, LINC01140, bone metastasis between the LINC01140 and an abundance of genes in samples from the BC study was investigated. These genes, such as chordin like 1 and bone morphogenic protein 6, participate in the development and progression of tumor growth and bone metastasis. Finally, the present study observed the interaction between microRNA (miR)-200b and miR-200c with LINC011440. The results from the present study indicated that higher expression of LINC01140 was beneficial for patients with BC. LINC01140 may be a potential biomarker for the prognosis of patients with BC. The role of LINC01140 in BC needs to be further evaluated.

\section{Introduction}

Breast cancer (BC) is one of the most common and dangerous malignant disorders among women. There are $\sim 1.7$ million new cases of BC every year, accounting for a quarter of all new cancer cases in women worldwide (1). There is also a higher rate of mortality and morbidity among patients with $\mathrm{BC}$, despite the rapid development of modern diagnostic and therapeutic techniques (2-4). Elucidating the molecular mechanisms underlying $\mathrm{BC}$ is essential for discovering new therapeutic targets for $\mathrm{BC}$. Therefore, understanding the regulatory network underlying $\mathrm{BC}$ is an essential focus point for future studies. There is an increasing amount of evidence that long non-coding RNAs (IncRNAs) participate in the development and progression of $\mathrm{BC}$ and potentially serve as markers of diagnosis or prognosis $(4,5)$.

LncRNAs are defined as RNA transcripts $>200$ bp in length that lack open reading frames. A number of lncRNAs were preliminary identified by whole-genome sequencing and next generation transcriptome sequencing (6). In previous years, numerous studies have demonstrated that IncRNAs play an important role in the biological process of various diseases, including carcinogenesis and tumor progression, particularly in $\mathrm{BC}$, by means of regulating gene expression at the epigenetic, transcriptional and post-transcriptional levels $(6,7)$.

Long intergenic non-protein coding RNA 01140 (LINC01140) is located on chromosome 1p22.3. At the site of transcription, LINC01140, unlike proteins, could accommodate the transcription of genome-adjacent protein-coding genes in cis and regulate the transcription of distal protein-coding genes in trans $(8,9)$. The association between LINC01140 expression and survival outcome was evaluated in patients with gastric 
A

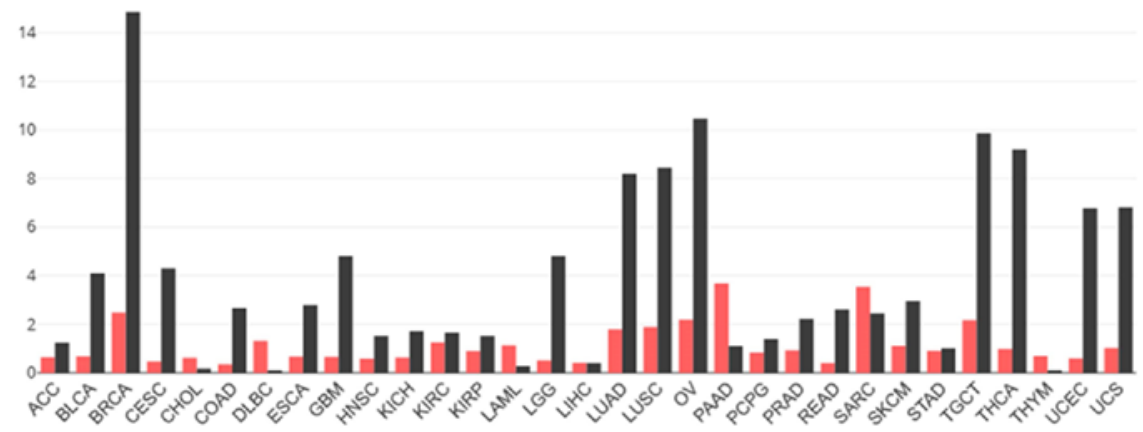

B

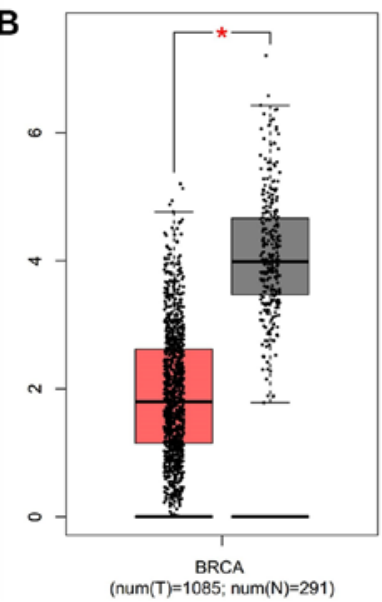

C

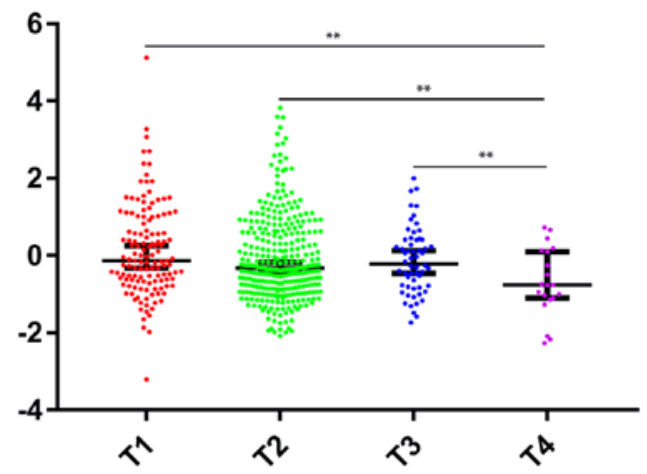

Figure 1. LINC01140 is downregulated in BC tissues. (A) The LINC01140 expression profile across all tumor samples and paired normal tissues. (B) LINC01140 expression was decreased in breast cancer compared with normal tissues. The height of bar represents the median expression level of certain tumor type (red) or normal tissue (black). (C) LINC01140 expression in breast cancer is associated with certain clinical pathological parameters in different stages. The 825 patients with breast cancer were grouped according to their tumor size. LINC01140 expression in each different group of stages is presented as the mean \pm standard error of the mean. A two-tailed Student's t-test was used to calculate statistical significance. ${ }^{*} \mathrm{P}<0.05$ and ${ }^{* *} \mathrm{P}<0.01$. LINC01140, long intergenic non-protein coding RNA 01140 .

cancer (GC). The results demonstrated that LINC01140 is significantly associated with overall survival in patients with GC (9). Therefore, it is valuable to investigate the functional roles of LINC01140. To investigate the roles and molecular mechanisms of LINC01140 in BC, LINC01140 expression was analyzed in 1,085 breast cancer patients and 291 healthy subjects using Gene Expression Profiling Interactive Analysis (GEPIA). The association between LINC01140 expression and T stages, LINC01140-related biological pathways, and the correlation between certain LINC01140 expression genes were also analyzed in 825 patients with $\mathrm{BC}$ via the cBioPortal database.

\section{Materials and methods}

The data on LINC01140 expression from 1,085 patients with $\mathrm{BC}$ and 291 healthy tissues were collected from the web resource of Gene Expression Profiling Interactive Analysis (GEPIA) (10). GEPIA is a developed interactive web server for analyzing RNA sequencing expression data from tumors and normal samples. The present study acquired the clinical data for the patients with BC from the open access cBioPortal database (http://www.cbioportal.org) $(11,12)$, which provides web resources for analyzing multidimensional cancer genome data, as well as graphical summaries of gene-level data from multiple platforms and survival analyses. The present study used a two-tailed Student's t-test to evaluate statistical discrepancies between two groups. Survival probability was determined using a Kaplan-Meier plotter (http://kmplot. com/analysis) (13). Patients with $\mathrm{BC}$ were divided into high LINC01140 or low LINC01140 based on the median value of LINC01140 expression. The association between LINC01140 expression and T stages, LINC01140-related biological pathways, and the correlation between LINC01140 expression genes were analyzed in 825 patients with $\mathrm{BC}$ through the cBioPortal database (14). Gene enrichment analyses were performed using FUNRICH software (version 3.0) for the co-expressed genes from the cBioPortal database (15). The microRNA (miRNA/miR)-LINC01140 interactions were analyzed by Ago CLIP-seq Data from starBase (http://starbase.sysu.edu.cn) (16).

\section{Results}

LINC01140 is downregulated in BC tissues and low LINC01140 expression is associated with a worse prognosis in patients with $B C$. In multiple types of cancer, particularly in BC, the expression of LINC01140 is downregulated (Fig. 1A and B). Compared with the mammary tissues in $\mathrm{BC}$, the expression of LINC01140 is almost 6 times higher in normal mammary tissues. In the present study, LINC01140 expression in 1,085 patients with BC and 291 healthy subjects from RNA sequencing datasets was analyzed through GEPIA. It was revealed that LINC01140 expression was significantly 

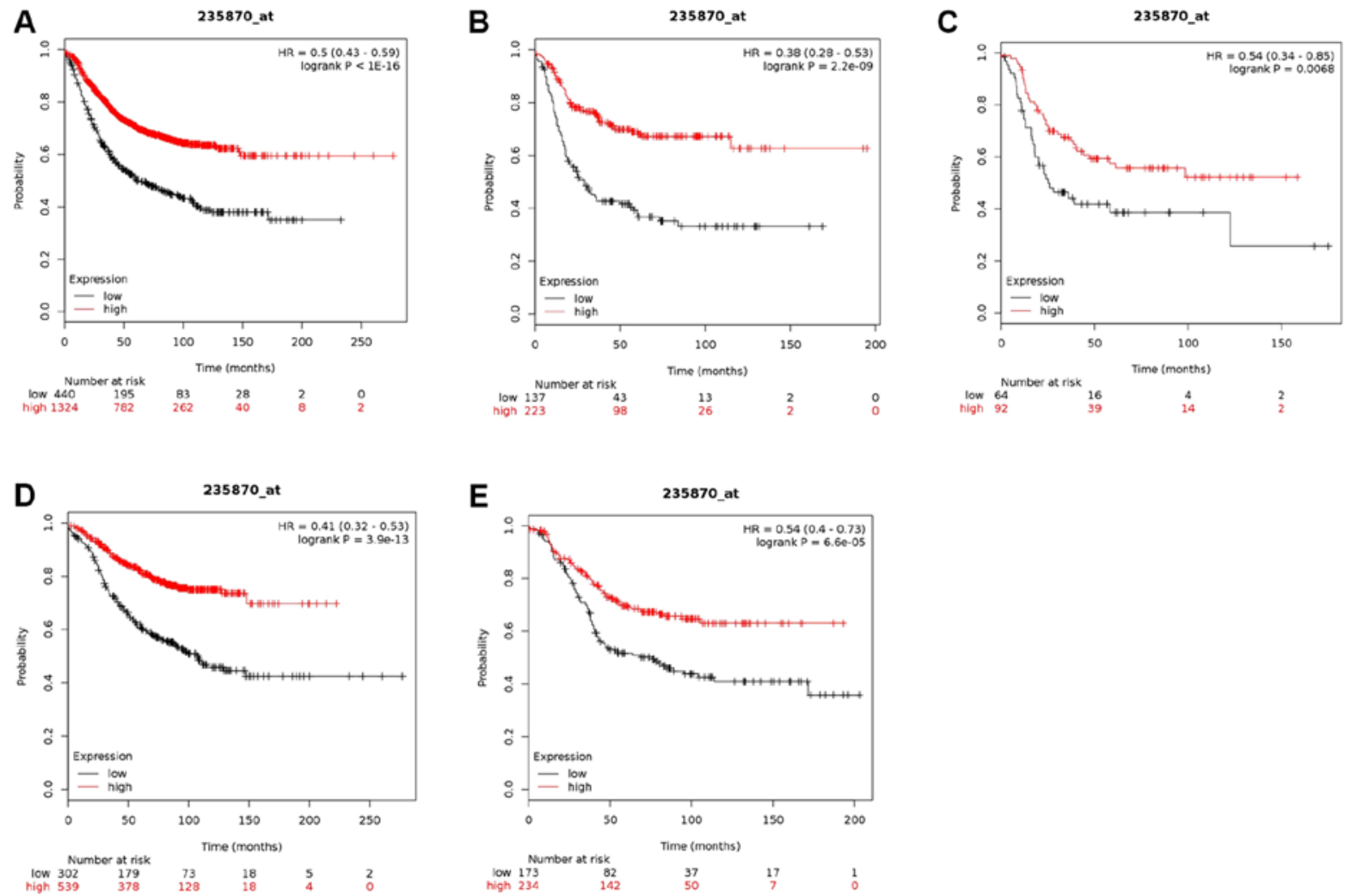

Figure 2. Impact of the LINC01140 (Affymetrix ID: 235870_at) expression on the survival probability and breast cancer. Survival probability determined by Kaplan-Meier plotter. Patients with breast cancer were divided into high expression of LINC01140 (red) or low expression of LINC01140 (black) in the light of the median value of LINC01140 expression. (A) Represents the RFS of all patients without distinguishing between breast cancer subtypes. RFS probability in the four subtypes: (B) Basal-like RFS, (C) HER2-positive RFS, (D) Luminal-A RFS and (E) Luminal B RFS. RFS, relapse-free survival; HR, hazard ratio LINC01140, long intergenic non-protein coding RNA 01140.

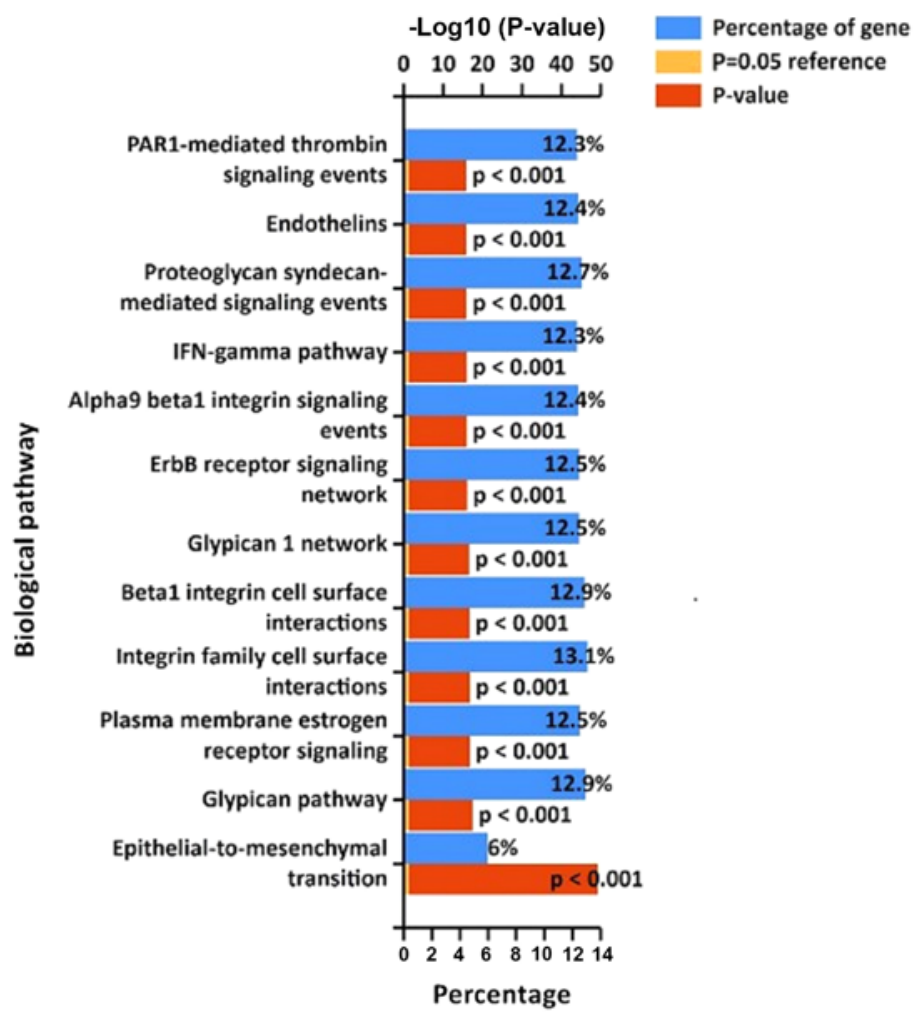

Figure 3. LINC01140 is involved in biological pathways. The biological pathways include PAR1-mediated thrombin signaling events, endothelins, proteoglycan syndecan-mediated signaling events, IFN- $\gamma$ pathway, $\alpha 9 \beta 1$ integrin signaling events, ErbB receptor signaling network, glypican 1 network, $\beta 1$ integrin cell surface interactions, intergrin family cell surface interactions, plasma membrane estrogen receptor signaling, glypican pathway and epithelial-to-mesenchymal transition. IFN, interferon; LINC01140, long intergenic non-protein coding RNA 01140. 
A
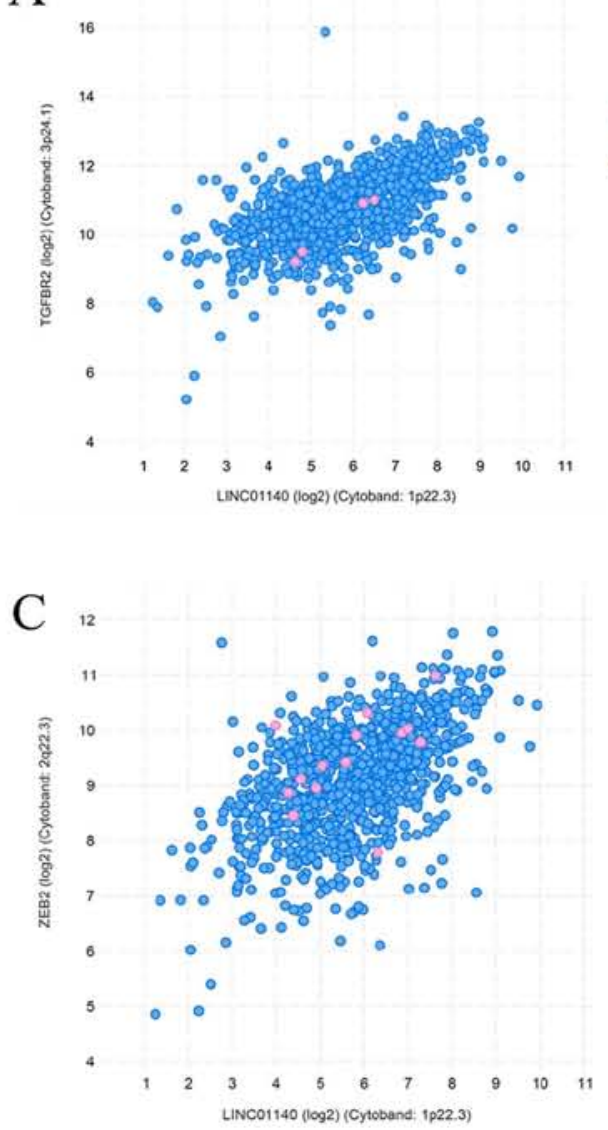

E 18

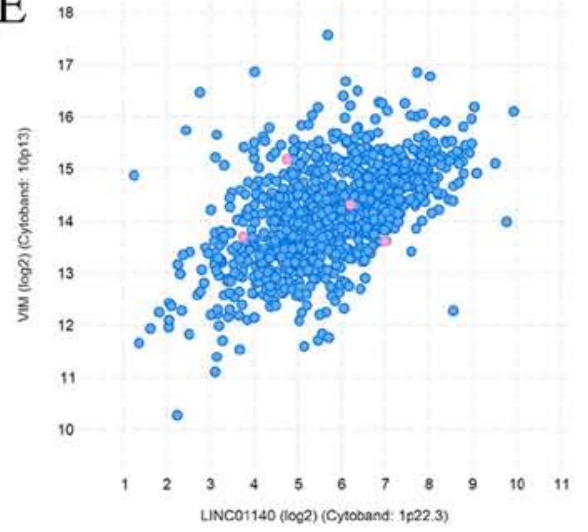

$\mathrm{G}$

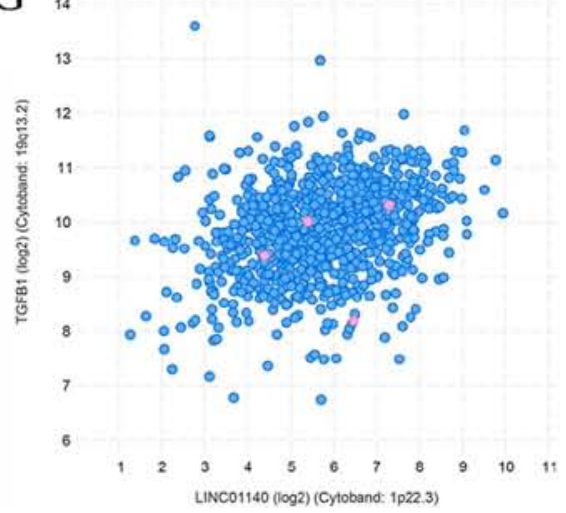

Pearson 0.58
Spearman: 0.59

- TGFBR2

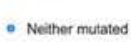

$\mathrm{B}$

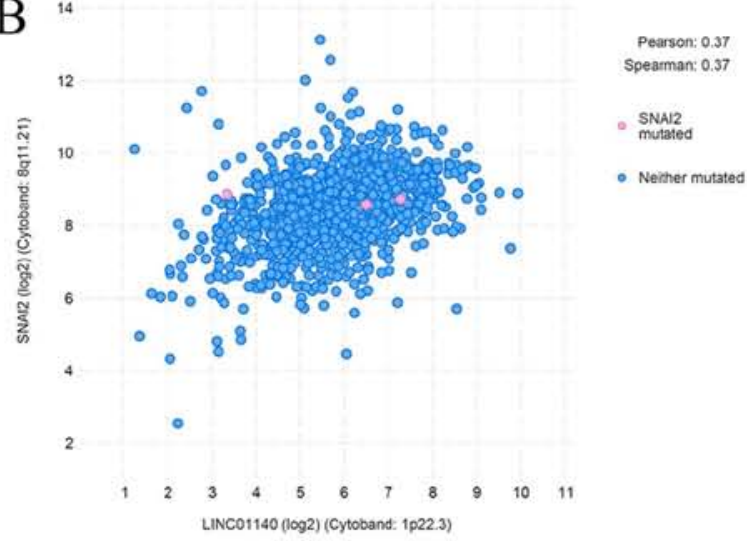

$\mathrm{D}$

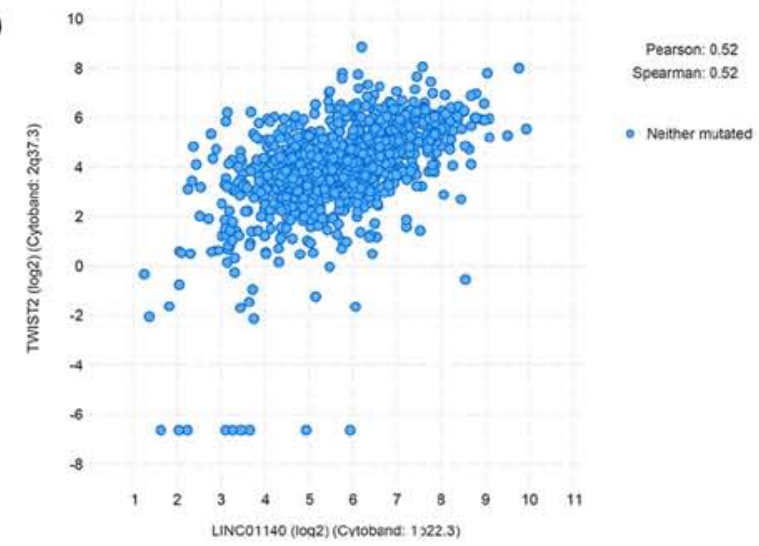

$\mathrm{F}$

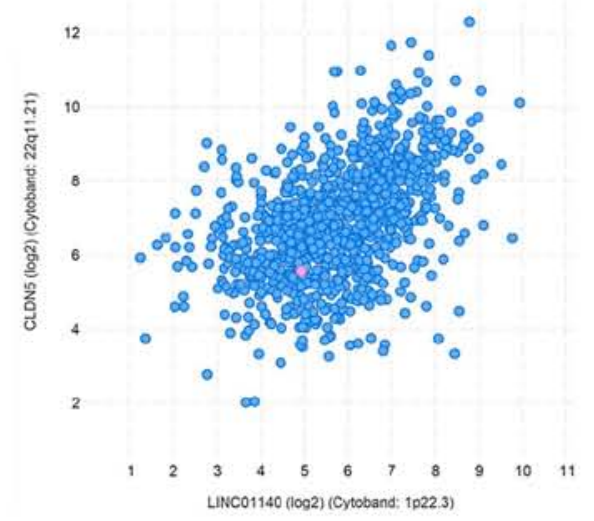

Pearson: 0.48

- CLON5
$\mathrm{H}$

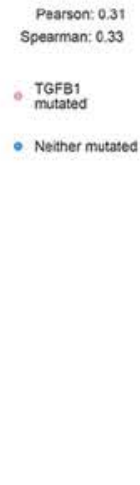

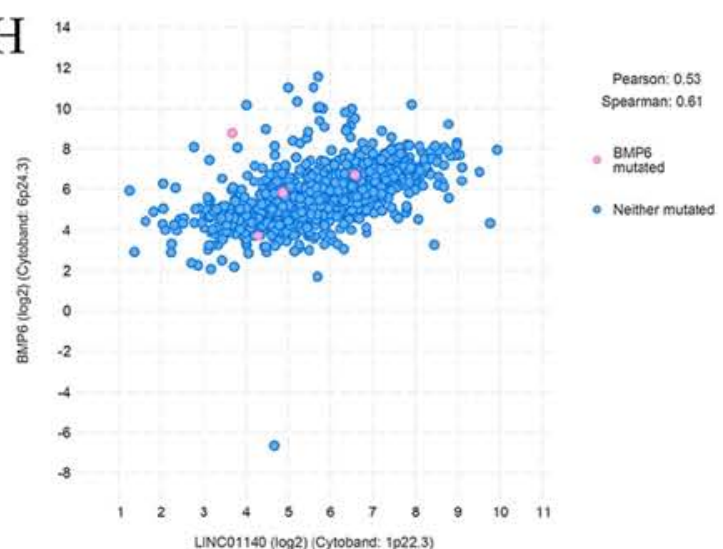

Figure 4. Plot showing the co-expression association between the LINC01140 and the abundance of genes in samples from the breast cancer study. These genes were co-expressed with LINC01140 expression. All the Pearson values are $>0.30$. (A) TGFBR2, (B) SNAI2, (C) ZEB2, (D) TWIST2, (E) VIM, (F) CLDN5, (G) TGFB1, (H) BMP6. 
I
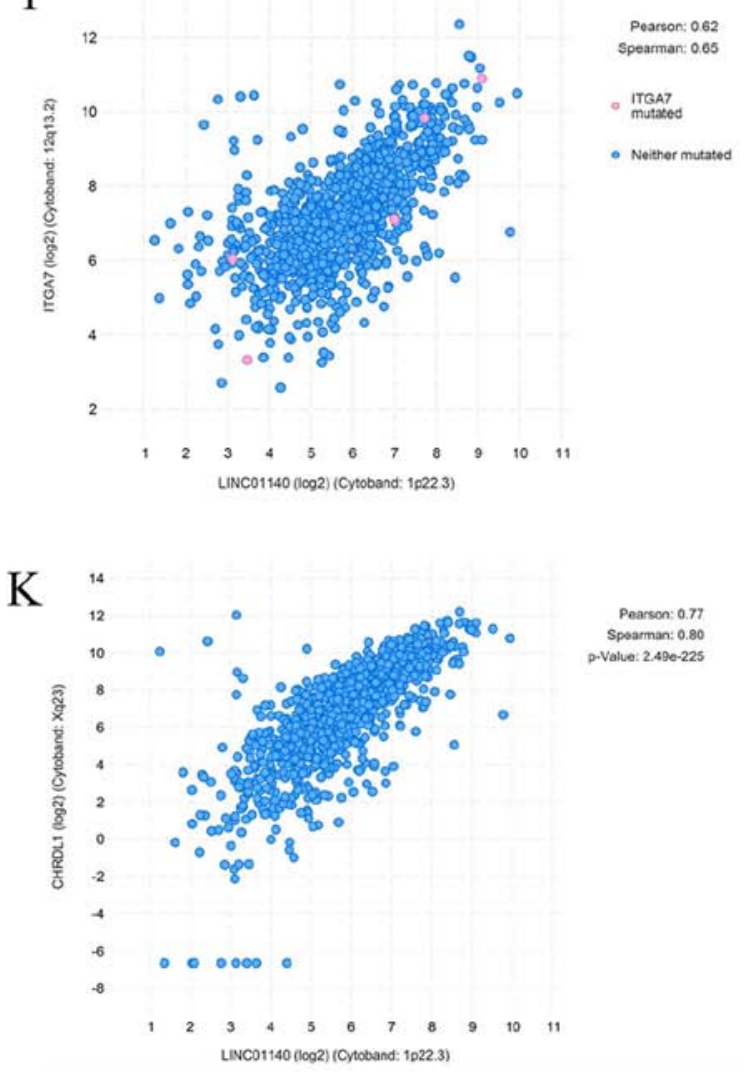

$\mathrm{J}$

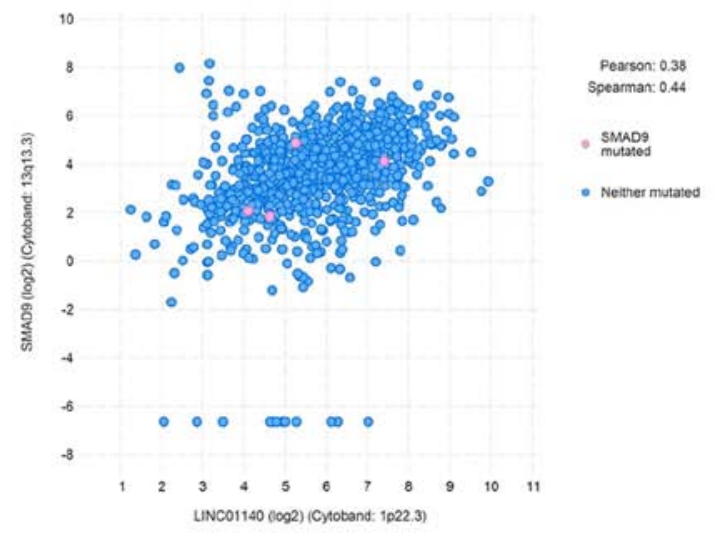

Figure 4. Continued. Plot showing the co-expression association between the LINC01140 and the abundance of genes in samples from the breast cancer study. These genes were co-expressed with LINC01140 expression. All the Pearson values are >0.30. (I) iTGA7, (J) SMAD9, (K) CHRDL1. LINC01140, long intergenic non-protein coding RNA 01140; TGFBR2, transforming growth factor $\beta$ receptor 2; SNAI2, snail family transcriptional repressor 2; ZEB2, zinc finger E-box binding homeobox 1; TWIST2, twist family bHLH transcription factor 2; VIM, vimentin; CLDN5, claudin 5; TGFB1, transforming growth factor $\beta 1$; BMP6, bone morphogenic protein 6; ITGA7, integrin subunit $\alpha$; SMAD9, SMAD family member 9; CHRDL1, chordin like 1.

decreased in $\mathrm{BC}$ tissues compared with normal tissues $(\mathrm{P}<0.05$; Fig. 1B). The impact of the LINC01140 expression on RFS probability and BC was analyzed using a Kaplan-Meier plotter. The results revealed that in general, high LINC01140 expression predicts longer RFS probability in all patients with BC, including those with Basal-like, HER2-positive and Luminal-A and Luminal-B subtypes. While, low LINC01140 expression levels were associated with shorter RFS survival probability of patients with BC (Fig. 2), which is consistent with the fact that LINC01140 expression was decreased in BC compared with normal tissues.

Expression of LINC01140 is associated with T stages of patients with $B C$. The 825 patients with $\mathrm{BC}$ were divided into T1, T2, T3 and T4 stages according to tumor size. The detailed demographic of the patients with BC is presented in Table SI. It was revealed that LINC01140 expression was significantly decreased in $\mathrm{T} 4$ stage in the patients with $\mathrm{BC}$ when compared with T1, T2 or T3 stages $(\mathrm{P}<0.01$; Fig. $1 \mathrm{C})$. Above all, it was demonstrated that higher LINC01140 expression was beneficial for patients with $\mathrm{BC}$.

Potential biological pathways or processes that LINC01140 may be involved in. The present study also demonstrated that LINC01140 may be involved in a series of biological pathways, such as PAR1-mediated thrombin signaling events, endothelins, proteoglycan syndecan-mediated signaling events, interferon- $\gamma$ pathway, $\alpha 9 \beta 1$ integrin signaling events, ErbB receptor signaling network, glypican 1 network, $\beta 1$ integrin cell surface interactions, integrin family cell surface interactions, plasma membrane estrogen receptor signaling, glypican pathway and particularly in the EMT (Fig. 3).

Results in Fig. 4 showed the correlation of each gene expression with LINC01140 expression, including transforming growth factor $\beta$ receptor 2 (TGFBR2; Pearson $\mathrm{r}=0.58)$, snail family transcriptional repressor 2 (SNAI2; Pearson $\mathrm{r}=0.37$ ), zinc finger E-box binding homeobox 1 (ZEB2; Pearson $\mathrm{r}=0.53)$, twist family bHLH transcription factor 2 (TWIST2; Pearson $\mathrm{r}=0.52$ ), vimentin (VIM; Pearson $\mathrm{r}=0.52)$, claudin 5 (CLDN5; Pearson $\mathrm{r}=0.48)$, transforming growthfactor $\beta 1$ (TGFB1; Pearson $\mathrm{r}=0.31$ ), bone morphogenic protein 6 (BMP6; Pearson $\mathrm{r}=.53)$, integrin subunit $\alpha 7$ (ITGA7; Pearson r=0.62), SMAD family member 9 (SMAD9; Pearson $\mathrm{r}=0.38)$ and chordin like 1 (CHRDL1; Pearson $\mathrm{r}=0.77)$. These genes were co-expressed with LINC01140 expression. The miRNA-LINC01140 interactions supported the Ago CLIP-seq Data. It was observed that miR-200c and miR-200b interacted with LINC01140 (Fig. 5). The competing endogenous RNA interaction network of LINC01140 in humans was also obtained through Ago CLIP-seq Data. LINC01140 and PITX2 share miRNA families, such as miR-200c and miR-200b (Table I). 


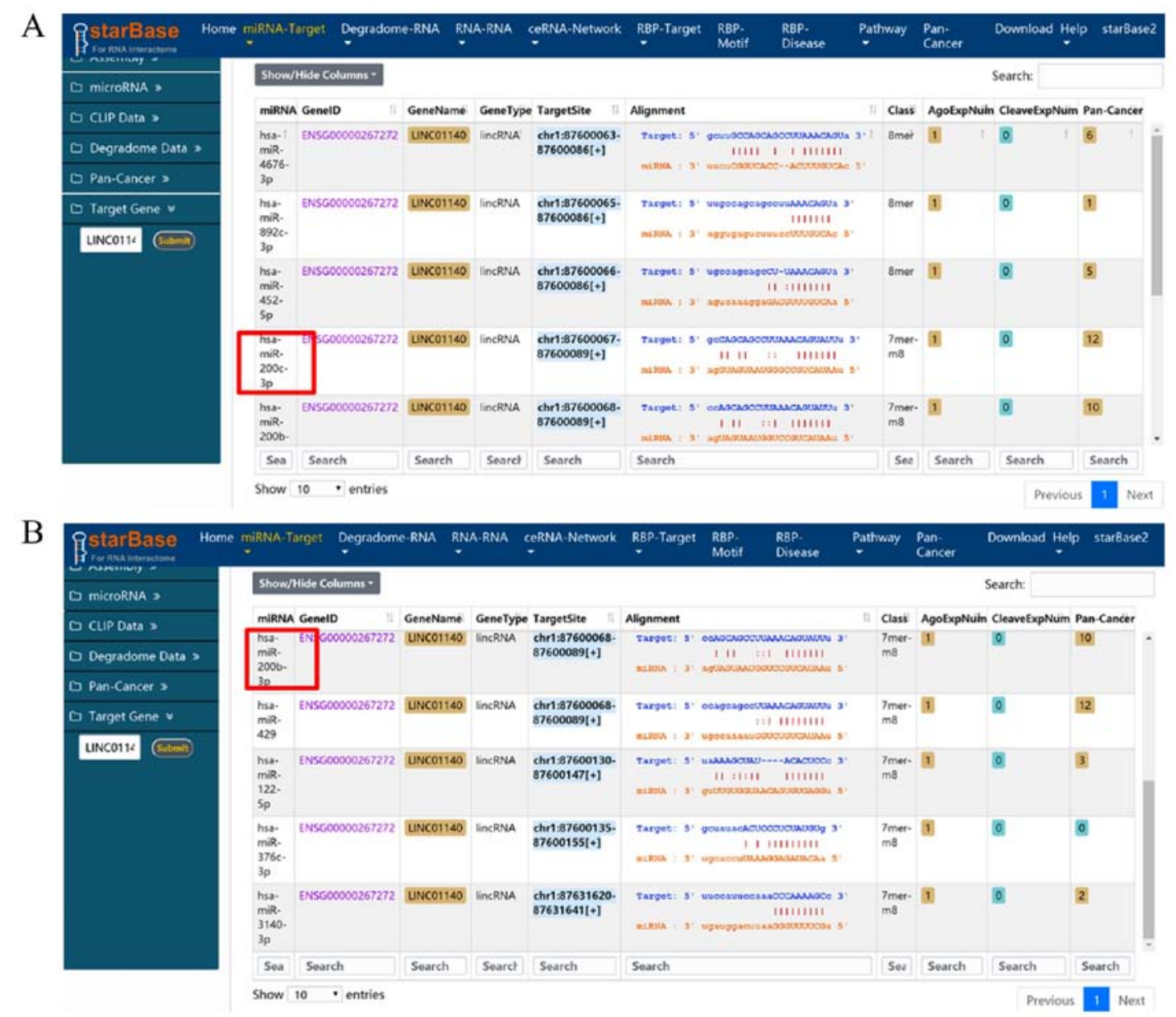

Figure 5. miRNA-LINC01140 interactions were supported by Ago CLIP-seq Data. (A) miR-200c and (B) miR-200b were demonstrated to interact with LINC01140. miRNA/miR, microRNA; LINC01140, long intergenic non-protein coding RNA 01140.

\section{Discussion}

LINC01140 is located in chromosome $1 \mathrm{p} 22.3$ and is involved in regulating the transcription of genomic neighboring protein coding genes and long distance protein-coding genes in cis or in trans, respectively. A previous study evaluated the association between LINC01140 expression and survival outcome in patients with GC (9). A significant correlation between LINC01140 and overall survival was observed in patients with GC. Therefore, LINC01140 was combined to form a single prognostic signature in GC.

The present study also revealed that LINC01140 is involved in numerous different biological pathways, particularly in the epithelial-to-mesenchymal transition (EMT). EMT is crucial for embryogenesis as a cellular program, which plays a role in malignant progression and wound healing. Numerous studies in humans have demonstrated that increased tumor-initiating and metastatic potential were promoted by EMT during neoplasia (17). EMT is controlled by a number of transduction pathways culminating in the core and dominant transcription factors of the process (18). Notably, the co-expression relationship between the LINC01140 and an abundance of genes in samples from the BC study was revealed. These genes encode the core transcription factors that play a vital role in the EMT, such as TGFBR2, SNAI2, ZEB2, TWIST2, VIM, CLDN5, TGFB1, BMP6, ITGA7, SMAD9 and CHRDL1.
CHRDL1 is a bone morphogenetic protein (BMP) inhibitor and is involved in modulating the activity of the BMP signaling pathway. The CHRDL1 protein could competitively bind to BMPs and antagonize their function by the means of secreting into the extracellular space (19). BMPs were first identified for inducing ectopic bone formation and play an important role in morphogenesis during the development period. They are multifunctional growth factors that belong to the superfamily of transforming growth factor- $\beta$ (TGF- $\beta$ ) (20). Cyr-Depauw et al (21) demonstrated that under decreased ShcA signaling conditions, CHRDL1 expression is upregulated in a large amount of breast cancer cells following TGF-stimulation. The authors also observed that CHRDL1 plays a role as an inhibitor of BMP4-induced migration and invasion by the way of in vitro experiments. Furthermore, CHRDL1 expression is a favorable prognostic factor for patients with BC. Another study revealed a significant downregulation of CHRDL1 expression in GC tissues, which was associated with a low survival rate. A close association between low CHRDL1 expression and metastasis was observed through the clinical and pathological parameter. Furthermore, the authors revealed that downregulation of CHRDL1 promoted the proliferation and migration of tumor cells through the BMP receptor II. In addition, the authors confirmed that CHRDL1 is a tumor suppressor gene that inhibits tumor growth and metastasis (22). 
BMP6 is closely associated with tumor differentiation and bone metastasis (23). Hamdy et al (24) revealed that BMP6 expression in primary malignant cancer was closely associated with bone metastasis. The result implied that BMP6 may be partly responsible for osteoblastic changes in prostate cancer secondary metastases. Taking previous studies into consideration, it can be confirmed that patients with ER+ BC are more likely to develop bone metastasis. These results confirmed that BMP6 plays a vital role in the development of ER+ BC bone metastasis (23-25).

Further analyses observed that miR-200b and miR-200c interacted with LINC01140. The miR-200 family consists of five members in humans. One cluster comprised of miR-200b, miR-200a and miR-429 on the chromosome one, while another cluster included two members, miR-200c and miR-141, which were located on chromosome 12. During tumorigenesis and development, the miR-200 family could regulate cell viability, cell division, cell cycle progression, differentiation, apoptosis, self-renewal, reversal of chemotherapy resistance and EMT (26). Abnormal expression of the miR-200 family is associated with the occurrence and development of BC $(27,28)$. A study by Yang et al (29) demonstrated that the miR200 family is actively associated with the EMT through targeting and inhibiting the expression of genes, such as ZEB1, ZEB2, $\beta$-catenin/Wnt signaling, while a number of transcription factors, including Snail, Slug, ZEB-1, ZEB-2 and Twist1, are associated with tumor invasion and metastasis. Therefore, LINC01140 may interact with miR-200 according to the prediction results of the database.

In conclusion, LINC01140 expression was observed to be lower in individuals with BC. In general, its expression was associated with the probability of RFS. High LINC01140 predicts longer RFS probability of patients with BC. LINC01140 plays an important role in the development and progression of BC. Further studies are required in order to elucidate the underlying molecular mechanisms. The present study provides the foundations for investigating the underlying molecular mechanism of LINC01140 in the development and progression of BC.

\section{Acknowledgements}

Not applicable.

\section{Funding}

The present study was supported by the National Nature Science Foundation of China (grant nos. 81572921 and 81802494).

\section{Availability of data and materials}

The datasets used and/or analyzed during the present study are available from the corresponding author upon reasonable request.

\section{Authors' contributions}

YC and XC participated in the conception and design of the study. DL and LL analyzed and interpreted the data. DL and LL drafted the manuscript, and YC and XC edited it critically. All authors gave final approval of the version to be published. 


\section{Ethics approval and consent to participate}

Not applicable.

\section{Patient consent for publication}

Not applicable.

\section{Competing interests}

The authors declare that they have no competing interests.

\section{References}

1. Torre LA, Bray F, Siegel RL, Ferlay J, Lortet-Tieulent J and Jemal A: Global cancer statistics, 2012. CA Cancer J Clin 65 87-108, 2015.

2. Fan LM, Strasser-Weippl KM, Li JM, St Louis JB, Finkelstein DMP, Yu KM, Chen WM, Shao ZM and Goss PEP: Breast cancer in China. Lancet Oncol 15: e279-e289, 2014.

3. Akram M, Iqbal M, Daniyal M and Khan AU: Awareness and current knowledge of breast cancer. Biol Res 50: 33, 2017.

4. Hu X, Liu Y, Du Y, Cheng T and Xia W: Long non-coding RNA BLACAT1 promotes breast cancer cell proliferation and metastasis by miR-150-5p/CCR2. Cell Biosci 9: 14, 2019.

5. Jiang Y, Du F, Chen F, Qin N, Jiang Z, Zhou J, Jiang T, Pu Z, Cheng Y, Chen J, et al: Potentially functional variants in lncRNAs are associated with breast cancer risk in a Chinese population. Mol Carcinogen 56: 2048-2057, 2017.

6. Liu Y, Sharma S and Watabe K: Roles of lncRNA in breast cancer. Front Biosci (Schol Ed) 7: 94-108, 2015.

7. Niknafs YS, Han S, Ma T, Speers C, Zhang C, Wilder-Romans K, Iyer MK, Pitchiaya S, Malik R, Hosono Y, et al: The lncRNA landscape of breast cancer reveals a role for DSCAM-AS1 in breast cancer progression. Nat Commun 7: 12791, 2016.

8. Batista PJ and Chang HY: Long noncoding RNAs: Cellular address codes in development and disease. Cell 152: 1298-1307, 2013.

9. Song P, Jiang B, Liu Z, Ding J, Liu S and Guan W: A three-lncRNA expression signature associated with the prognosis of gastric cancer patients. Cancer Med 6: 1154-1164, 2017.

10. Li J, Han L, Roebuck P, Diao L, Liu L, Yuan Y, Weinstein JN and Liang H: TANRIC: An interactive open platform to explore the function of lncRNAs in cancer. Cancer Res 75: 3728-3737, 2015.

11. Cerami E, Gao J, Dogrusoz U, Gross BE, Sumer SO, Aksoy BA, Jacobsen A, Byrne CJ, Heuer ML, Larsson E, et al: The cBio cancer genomics portal: An open platform for exploring multidimensional cancer genomics data. Cancer Discov 2: 401-404, 2012

12. Gao J, Aksoy BA, Dogrusoz U, Dresdner G, Gross B, Sumer SO, Sun Y, Jacobsen A, Sinha R, Larsson E, et al: Integrative analysis of complex cancer genomics and clinical profiles using the cBioPortal. Sci Signal 6: pl1, 2013.

13. Nagy Á, Lánczky A, Menyhárt $\mathrm{O}$ and Győrffy B: Validation of miRNA prognostic power in hepatocellular carcinoma using expression data of independent datasets. Sci Rep 8: 9227, 2018.

14. Cancer Genome Atlas Network: Comprehensive molecular portraits of human breast tumours. Nature 490: 61-70, 2012.
15. Pathan M, Keerthikumar S, Ang C, Gangoda L, Quek CYJ, Williamson NA, Mouradov D, Sieber OM, Simpson RJ, Salim A, et al: FunRich: An open access standalone functional enrichment and interaction network analysis tool. Proteomics 15: 2597-2601, 2015.

16. Li JH, Liu S, Zhou H, Qu LH and Yang JH: starBase v2.0: Decoding miRNA-ceRNA, miRNA-ncRNA and protein-RNA interaction networks from large-scale CLIP-Seq data. Nucleic Acids Res 42 (Database Issue): D92-D97, 2014.

17. Dongre A and Weinberg RA: New insights into the mechanisms of epithelial-mesenchymal transition and implications for cancer. Nat Rev Mol Cell Biol 20: 69-84, 2019.

18. Voutsadakis I: Epithelial-Mesenchymal Transition (EMT) and regulation of EMT factors by steroid nuclear receptors in breast cancer: A review and in silico investigation. J Clin Med 5: pii: E11, 2016.

19. Balemans W and Van Hul W: Extracellular regulation of BMP signaling in vertebrates: A cocktail of modulators. Dev Biol 250: 231-250, 2002.

20. Attisano L and Wrana JL: Signal transduction by members of the transforming growth factor-beta superfamily. Cytokine Growth Factor Rev 7: 327-339, 1996.

21. Cyr-Depauw C, Northey JJ, Tabariès S, Annis MG, Dong Z, Cory S, Hallett M, Rennhack JP, Andrechek ER and Siegel PM: Chordin-like 1 suppresses bone morphogenetic protein 4-induced breast cancer cell migration and invasion. Mol Cell Biol 36: 1509-1525, 2016.

22. Pei YF, Zhang YJ, Lei Y, Wu DW, Ma TH and Liu XQ: Hypermethylation of the CHRDL1 promoter induces proliferation and metastasis by activating Akt and Erk in gastric cancer. Oncotarget 8: 23155-23166, 2017.

23. Zhang M, Wang Q, Yuan W, Yang S, Wang X, Yan J, Du J, Yin J, Gao SY, Sun BC and Zhu TH: Epigenetic regulation of bone morphogenetic protein- 6 gene expression in breast cancer cells J Steroid Biochem Mol Biol 105: 91-97, 2007.

24. Hamdy FC, Autzen P, Robinson MC, Horne CH, Neal DE and Robson CN: Immunolocalization and messenger RNA expression of bone morphogenetic protein- 6 in human benign and malignant prostatic tissue. Cancer Res 57: 4427-4431, 1997.

25. Maki DD and Grossman RI: Patterns of disease spread in metastatic breast carcinoma: Influence of estrogen and progesterone receptor status. Am J Neuroradiol 21: 1064-1066, 2000.

26. Mekala JR, Naushad SM, Ponnusamy L, Arivazhagan G, Sakthiprasad V and Pal-Bhadra M: Epigenetic regulation of miR-200 as the potential strategy for the therapy against triple-negative breast cancer. Gene 641: 248-258, 2018.

27. Ning X, Shi Z, Liu X, Zhang A, Han L, Jiang K, Kang C and Zhang Q: DNMT1 and EZH2 mediated methylation silences the microRNA-200b/a/429 gene and promotes tumor progression. Cancer Lett 359: 198-205, 2015.

28. Zhang G, Zhang W, Li B, Stringer-Reasor E, Chu C, Sun L, Bae S, Chen D, Wei S, Jiao K, et al: MicroRNA-200c and microRNA-141 are regulated by a FOXP3-KAT2B axis and associated with tumor metastasis in breast cancer. Breast Cancer Res 19: 73, 2017.

29. Yang J and Weinberg RA: Epithelial-mesenchymal transition: At the crossroads of development and tumor metastasis. Dev Cell 14: 818-829, 2008.

This work is licensed under a Creative Commons Attribution-NonCommercial-NoDerivatives 4.0 International (CC BY-NC-ND 4.0) License. 